\title{
Experimental quantification and numerical simulation of unsteady flow conditions during free flight maneuvers of insects
}

\author{
Andrei Shishkin, Peter Schützner, Claus Wagner and Fritz-Olaf Lehmann
}

\begin{abstract}
This study aimed to numerically simulate aerodynamic forces produced by wing motion of small fruit flies maneuvering freely inside a flight chamber. The kinematic data were derived from high-resolution, high-speed video measurements, tracking fluorescent markers on head, body and wings of the animal. We constructed a geometrical model of the fly and applied the kinematic data to simulate the free flight. Based on the calculated velocity and pressure fields, we evaluated vorticity and flight forces. Our numerical simulation confirmed experimentally predicted lift enhancing mechanisms such as the leading edge vortex, rotational circulation and wake capture, and thus appears to be a potent tool to study the impact of body motion on forces and moments during the various forms of flight maneuvers.
\end{abstract}

\section{Introduction}

Flying insects are famous for their impressive and unexcelled maneuvering capabilities during territorial and chasing behaviors, rapid avoidance reactions and escape responses. Uncovering the underlying physical principles of these behaviors is a challenging task due to the high dynamics and complexity of unsteady aerodynamic force production in flapping flight. Over the past decade, the mechanisms of force production in insects have been the subject of some rigorous theoretical and experimental investigations, revealing several novel lift enhancing aerodynamic mechanisms such as the leading edge vortex, rotational circulation and wake capture (Dickinson et al 1999). The majority of these investigations used mechanical

Andrei Shishkin, Claus Wagner

Fluid Systems, Institute of Aerodynamics and Flow Technology, German Aerospace Center, Bunsenstr. 10,37073 Göttingen, Germany, e-mail: Andrei.Shishkin@dlr.de, Claus.Wagner@dlr.de

Peter Schützner, Fritz-Olaf Lehmann

Biofuture Research Group, Institute of Neurobiology, University of Ulm, Albert-Einstein-Allee 11, 89081 Ulm, Germany, e-mail: Peter.Schuetzner@uni-ulm.de, Fritz.Lehmann@uni-ulm.de 
wings flapped by a robotic apparatus (Dickinson et al 1999, Ellington et al 1996, Maybury and Lehmann 2004) or computational models (Kliss et al 1989, Wang 2000, Sun et al 2007), and was performed under hovering flight condition, in which the induced wake only depends on the wings' own motion.

In a freely flying animal, by contrast, the production of vorticity and the shedding of vortical structures in each stroke cycle depend on additional factors such as wake components produced in a preceding half stroke or preceding stroke cycles, flow components resulting from force generation of wings flapping in close distance, changes in fluid velocity at the wings due to the animal's body motion, and external disturbances in the surrounding air. Altogether, these components determine the instantaneous flow regime around a flapping insect wing and thus lift and drag production (Lehmann 2008, Sane 2003). Ramamurti and Sandberg (2007) investigated the significance of body motion on force production and frictional damping using three-dimensional computational fluid dynamics simulations (CFD). They applied their programming code to the rapid turning behavior in fruit flies, termed flight saccades. The authors found that the additional flow components due to body rotation produce pronounced drag on the flapping wings that limits turning speed and effectively brakes rotational movements (FCT, flapping counter torque). Similar results were reported for turning flight of birds (cockatoos, Hedrick et al 2007, 2009), highlighting that frictional damping coefficients for roll and yaw are 4 to 6 -fold higher in these animals than in airplanes.

The dominant role of frictional damping due to body motion further contrasted a previous study on rotational damping in which mechanical wings mimicked free flight kinematics of fruit flies during saccadic turning (Fry et al 2003). In the latter study, the authors ignored body motion during their physical simulation and thus falsely concluded that turning behavior is dominated by inertial moments of the animal body and not by frictional damping due to wing drag. Behavioral studies on tethered fruit flies flying in a virtual-reality flight simulator later demonstrated that low frictional damping overloads the visuallycontrolled feedback-loop during maneuvering flight, potentially causing body instabilities in free flight (Hesselberg and Lehmann 2007). The modifications in local flow condition at flapping wings, moreover, change with increasing advance ratio, which is the ratio between forward speed of the animal and wing flapping velocity. In fruit flies, this ratio varies between zero at hovering flight and approximately 0.53 at maximum forward velocity $\left(1.22 \mathrm{~ms}^{-1}\right.$, wing flapping velocity $=2.29 \mathrm{~ms}^{-1}$ ), altering magnitude and direction of local air flow at the different flight modes. Consideration of wing kinematics and body movements is thus a key factor to understand aerodynamic force production in freely maneuvering animals (Fontaine et al 2009, Ristroph et al 2009, Schützner and Lehmann 2007).

This book chapter focuses on research funded by the National-Priority-Program SPP1207 of the German Science Foundation, presenting an approach towards the fundamental question of how body motion in an insect alters wake structure and unsteady aerodynamic force production. This is demonstrated by high-speed video measurements on wing and body motion of freely maneuvering $1.2 \mathrm{mg}$ fruit flies 
Drosophila and simulation of flow conditions and forces using an elaborated threedimensional CFD modeling approach.

\section{Experimental approaches - high speed video tracking technology}

To reconstruct wing motion of freely maneuvering fruit flies Drosophila melanogaster, we developed an experimental setup that consists of a cylindrical free-flight arena, three high-speed video cameras, an infrared light laser trigger, an infrared light sensitive camera, and commercial software packages. In the following paragraphs, we summarize the main features of the experimental components and describe how we derived temporally resolved kinematics of wings and body in three dimensions using self-written software.

\subsection{Tracking of fluorescent markers in a free flight arena}

All experiments were conducted in a $62 \mathrm{~mm}$ wide cylindrical free-flight arena with $70 \mathrm{~mm}$ diameter. To allow the animals to visually orientate inside the arena, the cylinder was made from Plexiglas and surrounded by a back-light illuminated cyanblack random dot pattern (Fig. 1). The upper end of the flight chamber was closed with a glass plate but equipped with several small lateral holes. The holes were filled with foam, allowing the exchange of air between chamber and the environment. In the middle of the arena, we mounted an upright tube filled with moist tissue that served both as a water dispenser and a launch pad for the flies. Before placing the flies into the arena, we marked head, body, and wings with fluorescent dye during low temperature anesthetization of $4^{\circ} \mathrm{C}$ on a Peltier stage. Each marker had a mass of approximately $0.1 \mu \mathrm{g}$ and a diameter of approximately $200 \mu \mathrm{m}$. Since the mass of a fruit fly wing amounts to only $3 \mu \mathrm{g}$, we limited the number of wing markers to the wing tip and the trailing edge, in order to avoid wing bending due to inertial effects. To minimize motion blur of the fluorescent markers on the captured video images, a ring of 48 ultraviolet light-emitting diodes produced short $60 \mu$ s light pulses, which were synchronized with three high-resolution, high-speed video cameras. We scored the significance of UV-light on visual orientation and vision capability by scoring tethered flying flies in their ability to track a visual target inside a virtual-reality, closed-loop flight simulator. Although we measured a small degradation in performance during UV-light illumination, all flies were capable to actively control the visual object displayed inside the simulator.

The three high-speed cameras were mounted with an inter-camera angle of $120^{\circ}$ above the arena that allowed us to track flies inside a volume of approximately $9900 \mathrm{~mm}^{3}$ at $3500 \mathrm{~Hz}$ frame capture rate. At the fly's $200 \mathrm{~Hz}$ wing stroke frequency, the latter value yields a temporal resolution of approximately 17.5 video frames per 


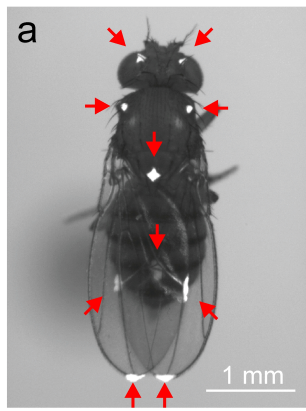

d

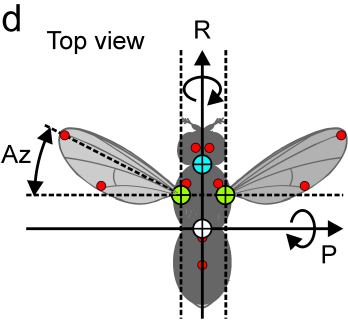

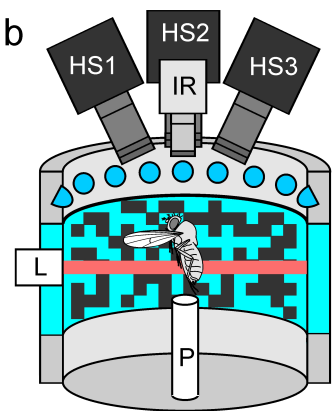

e

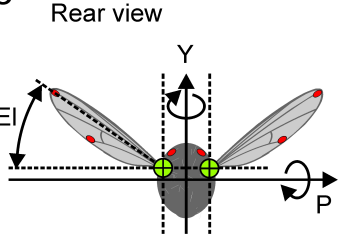

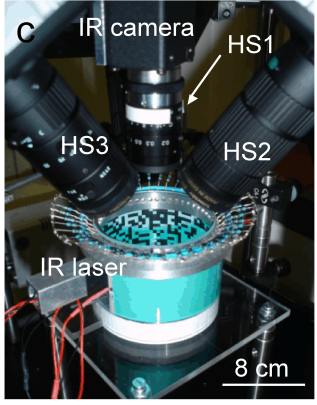

f

Side view

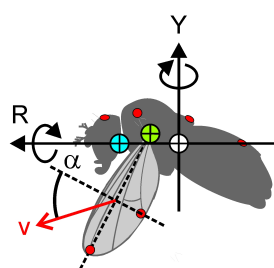

Fig. 1 Experimental setup for high-speed video recording in freely flying fruit flies. a Red arrows indicate fluorescent markers painted on head, body and wings of a fruit fly. b Sketch of the free flight arena. UV-light-emitting diodes (blue) stimulate fluorescent markers shown in a. HS, highspeed camera 1 to 3; IR, infrared sensitive camera; L, infrared laser; P, starting platform. Drawing is not to scale. c Image of the experimental setup. d-f Thorax axes and wing angles. Red dots indicate fluorescent markers. Center of body mass (white), center of head rotation (blue) and wing hinges (green) are derived from the fly's morphology. Az, wing azimuth; El, wing elevation; $\alpha$, wing's geometric angle of attack, with respect to wing velocity vector v; Y, vertical yaw axis; $\mathrm{P}$, horizontal pitching axis; R, horizontal roll axis.

wing stroke cycle or 9 frames per half stroke. The cameras were also equipped with optical filters matching the emitted orange-red light from the fluorescent markers and automatically triggered by the flying animal using an infrared light path. We constructed the light path employing a horizontally orientated infrared laser sheet, an infrared-sensitive camera and motion detector software. The camera lens was equipped with an infrared filter and a computer continuously recorded the infrared images with a frame rate of approximately $20 \mathrm{~Hz}$. Software analyzed the images online and generated a hardware trigger signal for the high-speed cameras whenever a fly crossed the laser sheet. To further increase the frequency of flight bouts within the recording time, we starved the flies one hour before placing them into the free flight arena, in which they remained up to 6 hours. 


\subsection{Three-dimensional reconstruction of wing motion}

The quality of computational models of flapping flight critically depends on the precision with which wing and body kinematics are measured in the freely maneuvering animal. It is less challenging to reconstruct markers on the fly body because those markers are comparatively large and only move at low speed during flight. By contrast, tracking azimuth and elevation angles of an oscillating wing including its angle of attack from small dye markers is more difficult and requires elaborated data processing. In particular, the precise reconstruction of fast kinematic maneuvers, such as speed and timing of wing rotation at the stroke reversals, is critical for force generation due to rotational circulation and wake capture enhancement (Dickinson et al 1999). Markers on wings may, moreover, be masked by the fly body or may disappear on the video images because of their small size and thus low fluorescence intensity. At the dorsal stroke reversal, fluorescent markers of tip and trailing wing edges even fuse during physical wing-wing contact, making the application of automatic tracking procedures more complicated (see second chapter of this book on insect flight).

We coped with the above problems by application of commercial video software that enhanced the visibility of the markers. The markers were subsequently tracked in each video frame using software developed in Matlab. To match corresponding video pixels in frames of all three high-speed video cameras, we applied a direct linear transformation algorithm, calibrated with a target at six distances from the cameras. The calibration target was mounted horizontally and displaced by a micromanipulator in $2.0 \mathrm{~mm}$ equidistant steps inside the measurement volume (Hedrick 2008). We repeated this procedure before and after the experiments to ensure high precision of the three-dimensional reconstruction.

The captured video frames were enhanced removing image noise and increasing image contrast by the box blur tool and image enhancement tool in VirtualDub, respectively. The image processing procedure conserved the brightness gradient from the border to the center of each marker that improved position tracking of the marker center. The tracking software routine was developed under Matlab and automatically scored the position of each marker according to the marker's center of area (Hedrick 2008). The algorithm provided sub pixel acuity of position estimates and greatly enhanced the quality of the data. Missing data points due to occlusion of fluorescent markers were interpolated applying separate spline functions to each coordinate value. The Matlab fit functions were also used for a 10-fold up sampling procedure of the position data. The latter step helped to smooth out high accelerations of the wing's rotational velocity during the stroke reversals due to the sparse number of data samples (2-3 data). During stroke reversals, the wing's angle of attack changes by approximately $90^{\circ}$ at an angular speed of approximately $90,000^{\circ}$ $\mathrm{s}^{-1}$.

We did not filter the wing position data (tips and trailing edges) because low pass filters produced pronounced motion artifacts in the data set. By contrast, we removed digitization noise from the body position data using a zero-phase onedimensional digital filter. It was applied separately for each $\mathrm{x} / \mathrm{y} / \mathrm{z}$ coordinate as an 
unweighted running average with a time window size of a quarter mean wing beat period (approximately $1.25 \mathrm{~ms}$ ).

We expanded the set of measured markers by a set of virtual body markers that were reconstructed from the fruit fly's morphology. From anatomical drawings (Demerec 1965), we estimated the position of the wing hinges, the fly's center of gravity, and the center of head rotation with respect to the three fluorescent markers on the thorax. The distances between these points were expressed in relative units and absolute values scaled according to the animal's size. We further reconstructed the longitudinal axis of wing rotation by a method developed in a side project of this SPP1207 investigation (Lehmann et al 2011). From the extended data set, we calculated various flight parameters such as yaw-, pitch- and roll angles of the body, horizontal and vertical flight direction, wing azimuth, elevation and angle of attack including their temporal derivatives in both the global and the fly body framework.

\section{Experimental results from 3D-reconstructions}
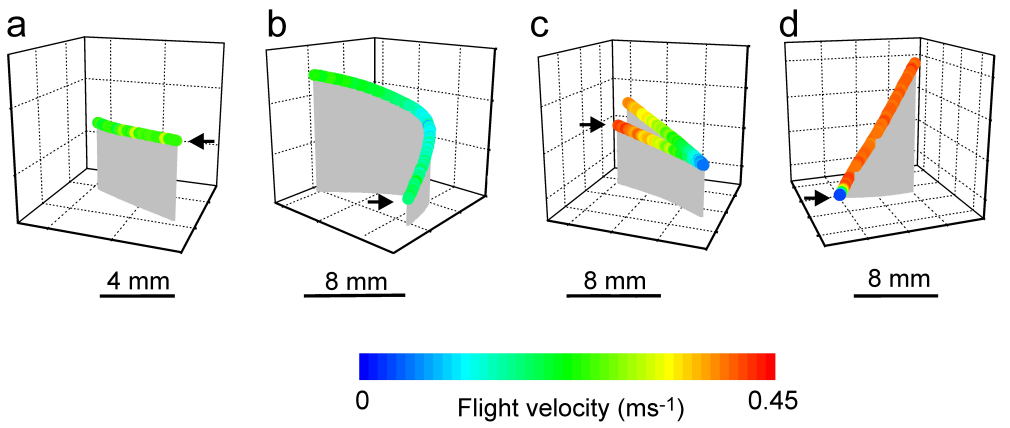

Fig. 2 Body motion (center of mass) during four types of flight maneuvers. a 28 ms sequence of level flight at low forward velocity. b $99 \mathrm{~ms}$ sequence of saccadic climbing flight. c $108 \mathrm{~ms}$ sequence of a backward/forward flight with reorientation maneuver during hovering. d $43 \mathrm{~ms}$ sequence of a takeoff maneuver. Arrow indicates flight start of the recorded path and grey area projects the flight trace into the horizontal.

Fig. 2 shows typical flight traces of four categories of flight maneuvers: level flight with constant forward velocity (Fig. 2a), a saccadic flight turn during climbing flight (Fig. 2b), backward flight with reorientation and subsequent forward acceleration (Fig. 2c), and take-off behavior from a starting platform (Fig. 2d). The data in Fig. 3 represent the corresponding wing and body motions underlying the flight sequence in Fig. 2a and have been used for the flow simulation described in the second part of this book chapter. Fig. 4 summarizes body velocity and orientation parameters of non-categorized flight behaviors from 170 fruit flies and flight sequences (32673 video frames), without scoring takeoff maneuvers. The histograms 
indicate that fruit flies prefer to fly at a mean forward flight speed ranging from 0.2 to $0.3 \mathrm{~ms}^{-1}$, but occasionally even fly backwards (negative values, Fig. 4a). Total flight speed is the vector sum of forward, sideward and vertical velocities and amounts to a maximum of approximately $0.7 \mathrm{~ms}^{-1}$. This value is equal to $57 \%$ of the maximum flight capacity estimated in flies flying freely under optomotor stimulation (Mronz and Lehmann 2008). Fig. 4e and f shows the relationship between forward velocity and yaw orientation of the longitudinal body axis of the animal. These data highlight the remarkable aerial capacity of fruit flies to independently control body orientation and flight direction. For example: data points that are lined up horizontally indicate flies changing flight direction, while keeping their yaw orientation constant. By contrast, data points that are lined up vertically indicate flight maneuvers in which flies rotate around their vertical axis without changing flight direction.

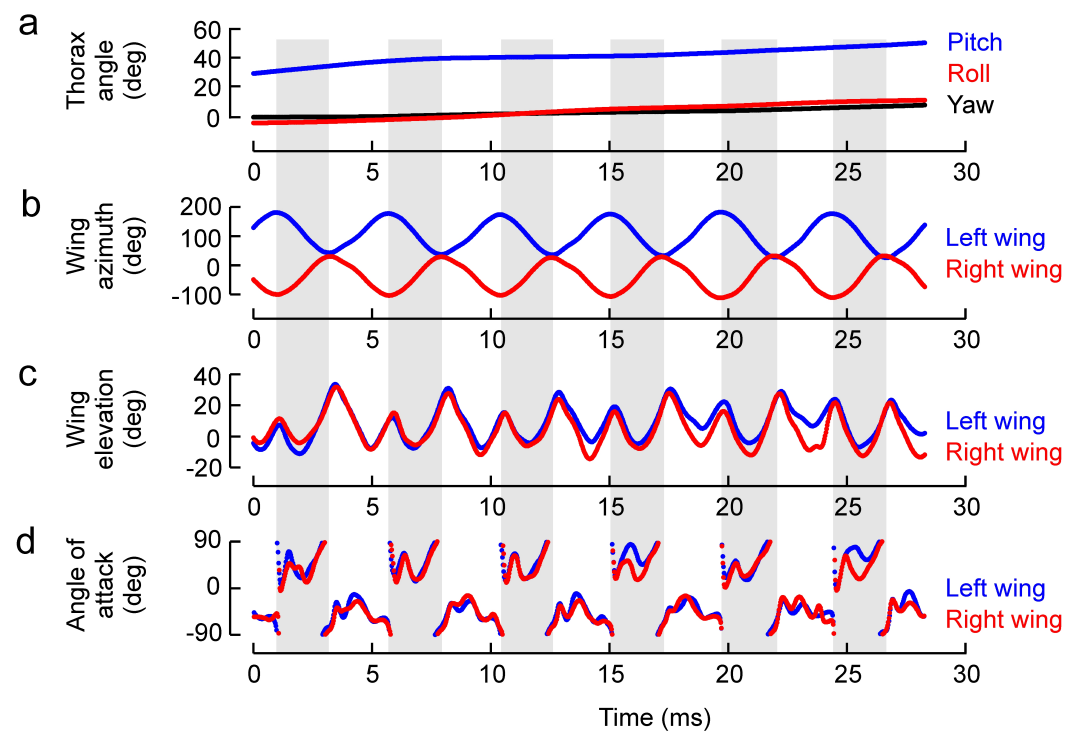

Fig. 3 Time-resolved body and wing angles of the flight sequence shown in Fig. 2a. a Yaw, pitch and roll angles of the thorax, where negative values indicate counter-clockwise (yaw, roll) or nosedown (pitch) angles. b Wing azimuth in the horizontal. c Wing elevation with respect to the vertical. d Geometric angle of attack of both wings with respect to the oncoming flow. This angle considers wing and body motion. The kinematic parameters (except for the angle of attack) are plotted with respect to the external (global) coordinate system. Grey area indicates the downstroke. 

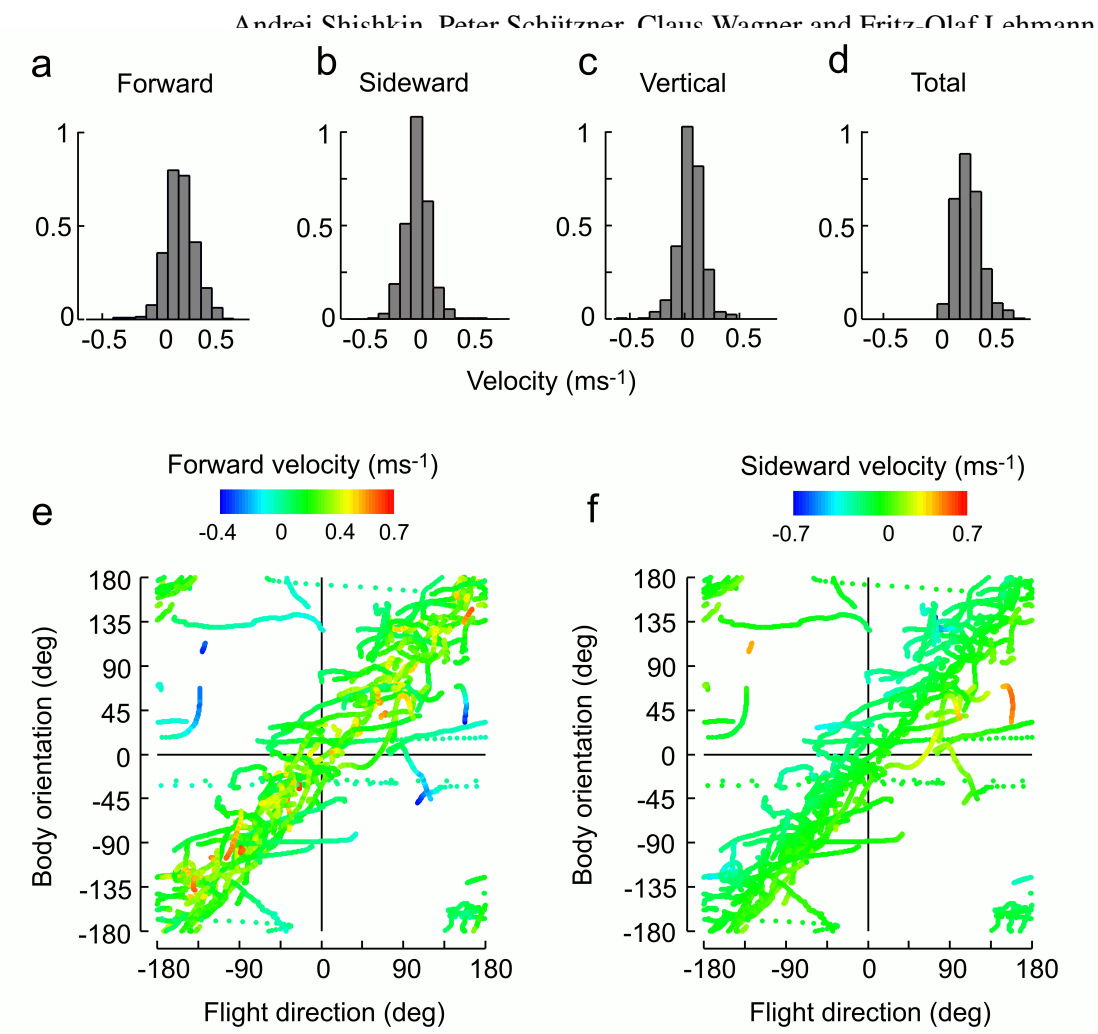

Fig. 4 Body dynamics in freely flying fruit flies. a-d Velocity distributions plotted as normalized histograms. Data show forward, sideward, and vertical velocity including their vector sum (total translational velocity). e-f Flight direction in the horizontal plotted against angular orientation (yaw) of the fly body. Data are plotted from 32673 video frames recorded during 170 flight sequences of approximately 170 flies.

\section{Numerical simulations of the free-flight induced flow}

A main objective of the presented study is to conduct computations of the flow around a freely flying fruit fly (Drosophila melanogaster) based on the measured kinematics plotted in Fig. 2a. A 3D-visualization of the experimentally obtained kinematics is depicted in Fig. 5, where the curves reflect the trajectories of the markers on the fly body and the wings. While the traces of the markers on the body show a nearly linear flight path, those located on the wing tips and trailing edges produce complicated curves (see Fig 5). 


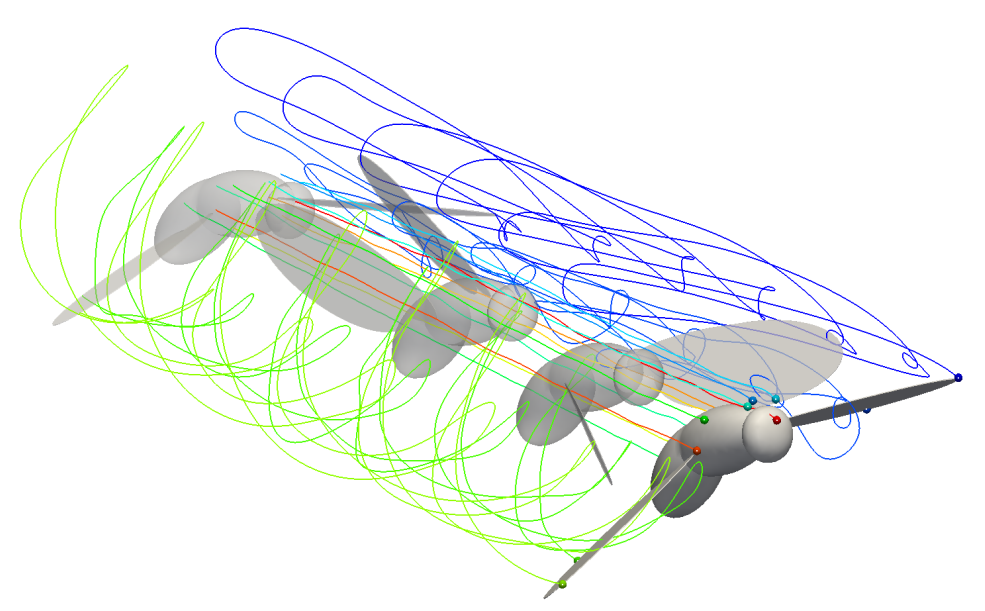

Fig. 5 Visualization of the Drosophila's free-flight kinematics used in the numerical simulations. The different lines show times traces of different markers painted on the fly's body and wings.

\subsection{Numerical method}

The Arbitrary Lagrangian Eulerian (ALE) formulation of Navier-Stokes equations allows to simulate flows in computational domains with moving boundaries. Expressed in terms of the dimensionless incompressible Navier-Stokes equations it reads:

$$
\frac{\partial \mathbf{u}}{\partial t}+\left(\left(\mathbf{u}-\mathbf{u}_{m e s h}\right) \cdot \nabla\right) \mathbf{u}=\frac{1}{R e} \nabla^{2} \mathbf{u}-\nabla p, \quad \nabla \cdot \mathbf{u}=0,
$$

where $\mathbf{u}$ and $p$ are the velocity and pressure fields, respectively, $R e$ is the Reynolds number and $\mathbf{u}_{\text {mesh }}$ is the mesh deformation velocity. Equations (1) depend on the time-dependent coordinates $\mathbf{x}(t)$ and the mesh velocity $\mathbf{u}_{m e s h}=\dot{\mathbf{x}}(t)$. Both functions are unknown but can be reconstructed using information from the moving boundaries. For each time step Eq. (1) is solved in steps with the following procedure:

$\mathbf{a}$ - find the smooth functions $\mathbf{x}(t)$ and $\mathbf{u}_{\text {mesh }}$

b - perform the mesh deformation using $\mathbf{x}(t)$ for a given time step and recalculate the fields needed for the step (c);

c - finally, solve the conventional Eulerian Navier-Stokes equations on the deformed mesh.

For the flow simulations presented below we used the standard ALE solver implemented in the open source OpenFOAM toolkit. The mesh motion velocity $\mathbf{u}_{\text {mesh }}$ needed for the step (a) is determined solving the Laplace equation:

$$
\nabla \cdot\left(\gamma \nabla \mathbf{u}_{m e s h}\right)=\mathbf{0},
$$


a

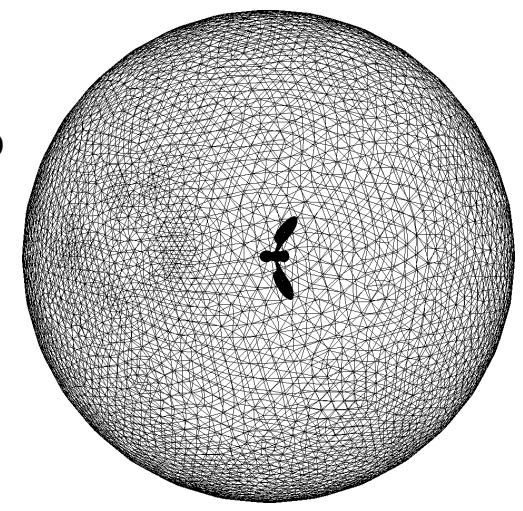

Fig. 6 Fruit fly model (a) and surface mesh of the computational domain (b).

in which the diffusivity factor $\gamma$ controls mesh deformation. The moving boundaries are the surfaces of the modeled fly body and the wings. Thus, the positions of the boundaries needed to solve Eq. (2) follow the fruit fly kinematics shown above.

To develop a geometric model suitable for the flow computations, the anatomical shape of the fruit fly, the measured kinematics and the computational requirements were taken into account. The resulting geometry of the fly model (Fig. 6a) consist of three solid parts: the first is the body, consisting of the head, the thorax and the abdomen, and the others are the two wings. With this approach, it was possible to simulate the measured kinematics of body and wings independently by using the same algorithm.

\subsection{Implementation of measured fruit fly kinematics}

The motion of a rigid body is defined through the coordinates $\mathbf{x}_{0}(t)$ of a point on the body and an orthogonal matrix $\mathbf{O}(t)$ which describes the rotation of the body around the center of rotation $\mathbf{x}_{0}(t)$. Thus, for any point $\mathbf{x}$ on the rigid body the equation of motion $\mathbf{x}(t)$ reads:

$$
\mathbf{x}(t)=\mathbf{x}_{0}(t)+\mathbf{O}(t)\left(\mathbf{x}\left(t_{0}\right)-\mathbf{x}_{0}\left(t_{0}\right)\right)
$$

where $t_{0}$ is a certain fixed time, $\mathbf{x}_{0}$ is a fixed point on the body and $\mathbf{O}(t)\left(=\mathbf{O}\left(\mathbf{x}_{0}, t\right)\right)$ is an orthogonal matrix, which does not depend on $\mathbf{x}$. By substitution of $t$ and $t_{0}$ by $t_{n+1}$ and $t_{n}$, respectively, subtraction of $\mathbf{x}\left(t_{n}\right)$ from Eq. (3), and after dividing by $\Delta t=t_{n+1}-t_{n}$, we obtain the Dirichlet boundary conditions for Eq. (2):

$$
\mathbf{u}_{m e s h}^{(n+1)}(\mathbf{x})=\frac{\mathbf{x}^{(n+1)}-\mathbf{x}^{(n)}}{t_{n+1}-t_{n}}=\frac{\mathbf{x}_{0}^{(n+1)}-\mathbf{x}^{(n)}+\mathbf{O}^{(n+1)}\left(\mathbf{x}^{(n)}-\mathbf{x}_{0}^{(n)}\right)}{t_{n+1}-t_{n}},
$$

where the superscript ${ }^{(n)}$ stands for the quantities taken at time $t_{n}$. 
To determine the time dependent vector $\mathbf{x}_{0}$ and the matrix $\mathbf{O}$ for each solid part of the model we used the kinematics of the three markers on each part. For example, considering that $\mathbf{x}_{b}(t), \mathbf{x}_{t}(t)$ and $\mathbf{x}_{e}(t)$ are the time series of the coordinates of the wing base, wing tip and wing edge markers, respectively, we take $\mathbf{x}_{0}=\mathbf{x}_{b}$. Furthermore, we construct an orthonormal basis of vectors $\left\{\mathbf{e}_{i}(t), i=1,2,3\right\}$ using the Gram-Schmidt orthogonalization of the sequence $\mathbf{x}_{e}-\mathbf{x}_{b}, \mathbf{x}_{t}-\mathbf{x}_{b}$ and $\left(\mathbf{x}_{e}-\mathbf{x}_{b}\right) \times\left(\mathbf{x}_{t}-\mathbf{x}_{b}\right)$. We consider the matrix $\mathbf{E}(t)=\left(\mathbf{e}_{1}(t), \mathbf{e}_{2}(t), \mathbf{e}_{3}(t)\right)$ comprised by the vectors $\left\{\mathbf{e}_{i}(t) ; i=1,2,3\right\}$ and the matrix

$$
\mathbf{O}(t)=\mathbf{E}(t) \mathbf{E}\left(t_{0}\right)^{T},
$$

where $t_{0}$ denotes the initial time, and the superscript ${ }^{T}$ stands for matrix transposition. Due to orthogonality of the unit vectors $\mathbf{e}_{i}(t), i=1,2,3$, the matrix $\mathbf{O}(t)$ is orthogonal, i.e. $\mathbf{O O}^{T}=\mathbf{I}$, where $\mathbf{I}=\left(\sigma_{i, j}\right)$ is the identity matrix. The identity in Eq. (3) can be proven by straightforward calculations (it is sufficient to validate the expression for $\left.\mathbf{x}=\mathbf{x}_{0}+\mathbf{e}_{i}, i=1,2,3\right)$. Thus, the vector $\mathbf{x}_{0}(t)$ and the matrix $\mathbf{O}(t)$ needed for the mesh motion boundary conditions (4) are constructed.

The boundary conditions Eq. (4) force the points on the moving surfaces to keep their local positions on the surface during the motion. Another type of the mesh motion boundary conditions is represented by the following equation:

$$
\left(\mathbf{u}_{m e s h}^{(n+1)}(\mathbf{x})-\frac{\left.\mathbf{x}_{0}^{(n+1)}-\mathbf{x}^{(n)}+\mathbf{O}^{(n+1)}\right)\left(\mathbf{x}^{(n)}-\mathbf{x}_{0}^{(n)}\right)}{t_{n+1}-t_{n}}, \mathbf{n}^{(n+1)}(\mathbf{x})\right)=0,
$$

where $(\cdot, \cdot)$ is the scalar product, $\mathbf{n}^{(n+1)}(\mathbf{x})=\mathbf{O}^{(n+1)} \mathbf{n}^{(n)}(\mathbf{x})$ is the surface normal at point $\mathbf{x}$. Under the conditions Eq. (6) the mesh motion velocity may have an additional component tangential to the surface. In other words, surface points can "slip" on the surface. These boundary conditions are more flexible than those defined by Eq. (4) and resist high mesh deformation, but they can be applied only to smooth surfaces.

Both types of mesh motion boundary conditions described above were implemented and used as appropriate.

\subsection{Computational details}

To conduct numerical simulations of incompressible flow induced by a fruit fly during free flight, we used a second order accurate finite volume method that solves the incompressible Navier-Stokes equations on unstructured grids. The measured kinematics were transformed to mesh motion boundary conditions (as discussed above) and the spherical computational domain of radius $R=20 \mathrm{~mm}$ shown in Fig. 6b was defined. The Drosophila model is located in the center of this sphere moving with the flight speed of the fly's center of gravity. The wing length of the fruit fly model 
a
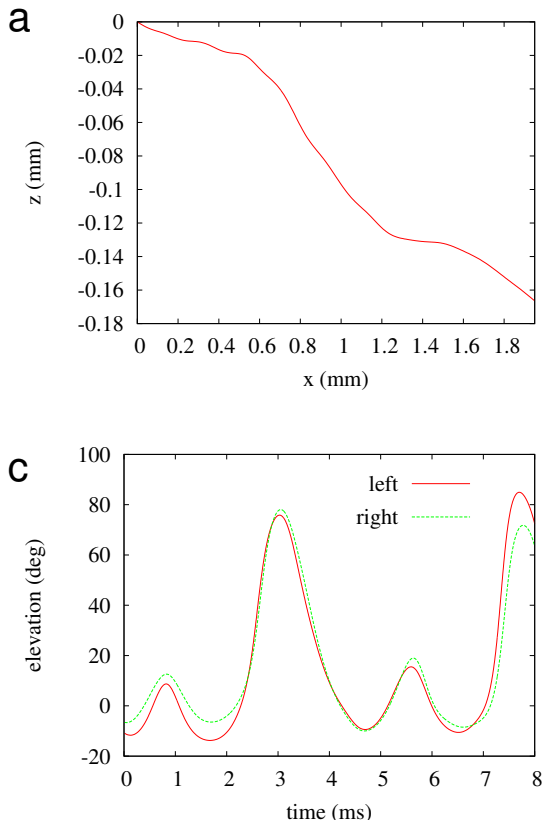
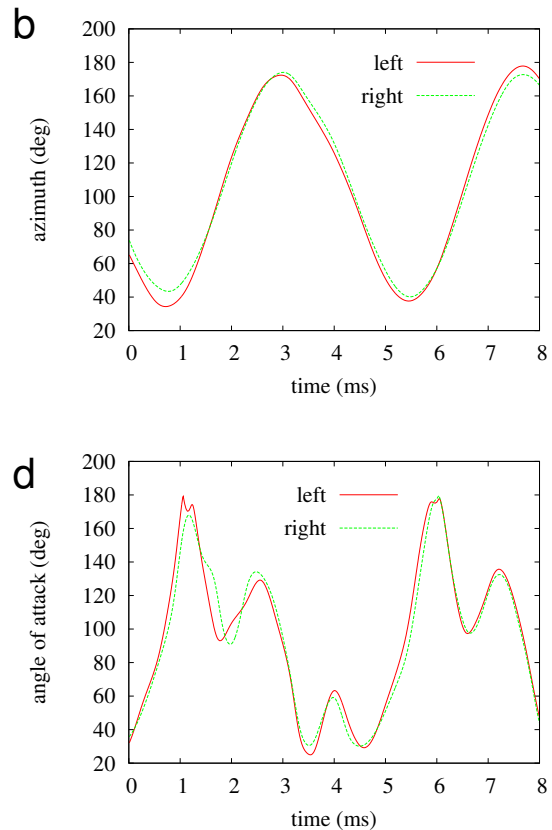

Fig. 7 Kinematics used in the numerical simulations. a The trace of the center of gravity of a fruit fly in $x z$-plane. b Azimuth, $\mathbf{c}$ elevation and $\mathbf{d}$ angles of attack of the two wings.

equals $\approx 2.5 \mathrm{~mm}$. Characteristic wing length and wing velocity yielded a Reynolds number of approximately 130.

Due to the high amplitudes of the wing strokes depicted in Fig. 5, it is not possible to simulate the measured fruit fly maneuvers by deforming a single moving mesh. After a certain number of time steps the mesh deformation produces some degenerated mesh cells that prevent an accurate solution of the Navier-Stokes equation. To overcome this, new meshes were occasionally generated and the flow fields of the previous time step were interpolated on these new meshes before the simulation was continued. The meshes with between 3.5 and $5 \cdot 10^{6}$ tetrahedral cells were generated with the open source mesh generator Netgen and the number of meshes needed for one wing stroke varied between 25 and 30 .

So far only the flow simulation for which a disembodied Drosophila model was assumed, covers more than one complete wing stroke. In this simulation the body kinematics define the motion of the computational domain while the wings follow the measured wing kinematics. The simulated time interval of $8 \mathrm{~ms}$ covers more than 1.5 wing strokes. For simplicity the data of the measured kinematics were orthogonally transformed such that the $z$-axis remains directed opposite to the gravity force, while the center of gravity of the fruit fly is initially placed at the origin of the coordinate system. Thus, during the simulated flight the center of gravity moves forward-and-down along the $x z$-plane with small fluctuations in $y$-direction as shown 
in Fig. 7a. The other parameters presented in Fig. 7 are the azimuth, i.e. the angle between the projection of wing base-tip vector on the (horizontal) $x y$-plane and the positive $x$-direction, the elevation (reflecting the angle between the base-tip vector and the $x y$-plane) and the angle of attack (AoA) which is the angle between the wing plane and $x y$-plane. These parameters slightly differ from the respective measured parameters due to some simplification made for the sake of being able to conduct flow simulations and, particularly, the use of a solid Drosophila model.

Since the local maxima and minima of the azimuth in Fig. $7 \mathrm{~b}$ define the start of each half strokes, the presented time interval can roughly be divided into the following phases: the time segment 0 to $1 \mathrm{~ms}$ corresponds to the downstroke, 1 to $3 \mathrm{~ms}$ - to the upstroke, 3 to $5.5 \mathrm{~ms}$ - to the downstroke and 5.5 to $7.5 \mathrm{~ms}$ - to the upstroke again. Variations in elevation angle and, even more, the angles of attack (see Fig. 7, c and d, respectively) are more complex reflecting local extrema within the half stroke intervals. This is mainly due to the changing angular velocities during wing rotation, which peak at $1 \mathrm{~ms}, 1.7 \mathrm{~ms}$, and $2.5 \mathrm{~ms}$.

\section{Free-flight induced flow fields}

Fig. 8 shows streamlines calculated at different times during the upstroke (1.7$3 m s$ and $6.8-8 m s$ ) and downstroke phase. The data suggest rather simple flow structures at the end of the upstroke $(0-3 \mathrm{~ms})$. By contrast, the flow becomes more vortical at $4.2 \mathrm{~ms}$, when the upstroke-induced flow structures start to interact with the vortices generated during the downstroke. As a consequence, the dynamic shedding of trailing edge vortices and wing-wake interactions generate a highly complex flow field in flapping flight.

The streamlines of the velocity vectors projected on a selected plane are presented in Fig. 9. Although the 2D graphs do not show the full complexity of the flow, they give an impression of the developing flow structure. The isosurfaces of pressure and vorticity shown in Figs 10 and 11 suggest that the developing flow during the upstroke affects the surrounding fluid only in the vicinity of the wings, where the isosurfaces of the pressure and vorticity magnitude are concentrated. At $t=3 m s$ a wake region starts to develop downstream the wings. During downstroke (up to $t \approx 0.55 \mathrm{~ms}$ ) stable regions characterized by higher and lower pressure values move with the wings. At the first half of downstroke (Fig. 9, $t=4.2 \mathrm{~ms}$ ), trailing edge vortices are shed, while the large pressure gradients produced in the wake region persist. They are later reused during the upstroke (Fig. 9, $t=6.8 \mathrm{~ms}$ to $8 \mathrm{~ms}$ ). The vorticity isosurfaces (Fig. 11, $t=4 \mathrm{~ms}$ and $t=5 \mathrm{~ms}$ ) also highlight regions with high vorticity and low pressure being attached to the leading edges of the wings (Fig. 12). LEV shedding is not observed at the down- upstroke transition in Fig. 9. We suppose that the complex wing kinematics, especially the changes in angle of attack, prevents LEVs from being shed during the stroke reversals.

Moreover, we calculated aerodynamic forces $\mathbf{F}$ acting on the wings which can be split into the pressure and shear components as follows: 



Fig. 8 The computed flow around the fruit fly visualized with 3D streamlines for different moments during the stroke cycle.

$$
\mathbf{F}=\underbrace{-\rho \iint_{S} p \mathbf{n} d S}_{\text {pressure component }}+\underbrace{\iint_{S} \mu\left[\nabla \mathbf{u}+(\nabla \mathbf{u})^{T}\right] \cdot \mathbf{n} d S}_{\text {shear component }},
$$

where $S$ denotes the wing surface with normal $\mathbf{n}$, and $\rho$ and $\mu$ are the density and the dynamic viscosity of air, respectively. The pressure force component is directed orthogonal to the wing plane, while the shear component acts tangentially. For further analysis each force component is decomposed by projection on vertical (z), forward $(x)$ and side $(y)$ directions to determine the lift, thrust and side forces, respectively.

Fig. 13 shows the resulting aerodynamic lift and thrust as pressure and shear components. The data indicate that pressure always exceeds shear forces during wing flapping. Nevertheless, the shear forces are of the same order as the weight of the Drosophila estimated as $\sim 10^{-6} \mathrm{~N}$.

The pressure contributions on lift and thrust shown in Fig. 13 are correlated with the elevation angles and the angles of attack. Lift and thrust transiently increase at the stroke reversals at $1-1.5 \mathrm{~ms}$ and $6-6.5 \mathrm{~ms}$, when rotational acceleration of the wings is maximum.

At times $1.5-3.0 \mathrm{~ms}$ and $6.5-8.0 \mathrm{~ms}$, the forces do not change much, while during the down stroke at $4.0-6.0 \mathrm{~ms}$ (Fig. 13) lift decreases and thrust increases. 

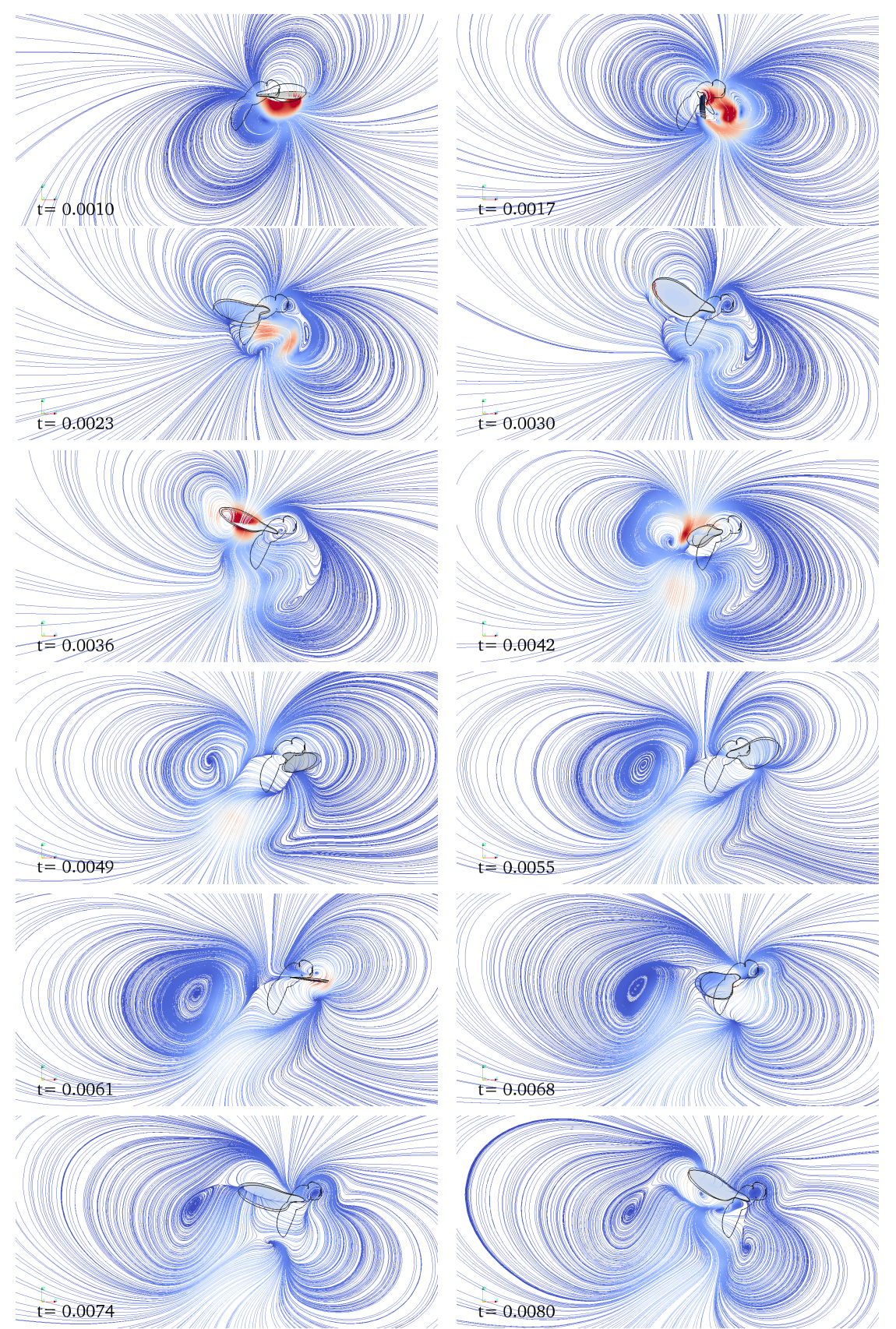

Fig. 9 2D view of the predicted streamlines around the fruit fly wing at different times of the stroke cycle. 

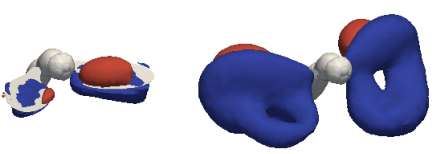

$\mathbf{t}=0.002 \mathrm{sec}$

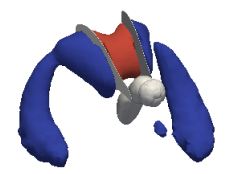

$t=0.003 \mathrm{sec}$

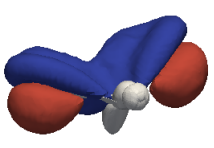

$t=0.004 \mathrm{sec}$

$\mathrm{t}=0.001 \mathrm{sec}$

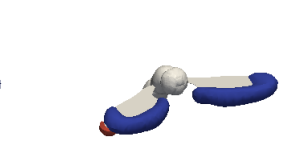

$t=0.005 \sec$

$\mathbf{t}=0.006 \mathrm{sec}$
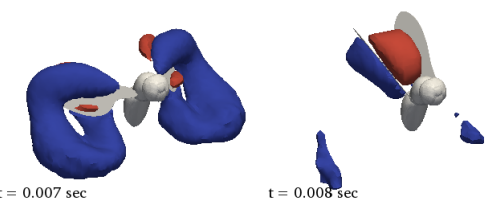

Fig. 10 Predicted isosurfaces of lower (blue) and higher (red) relative pressure field ( $p \sim \pm 10 \mathrm{~Pa}$ ) for different times of the stroke cycle.

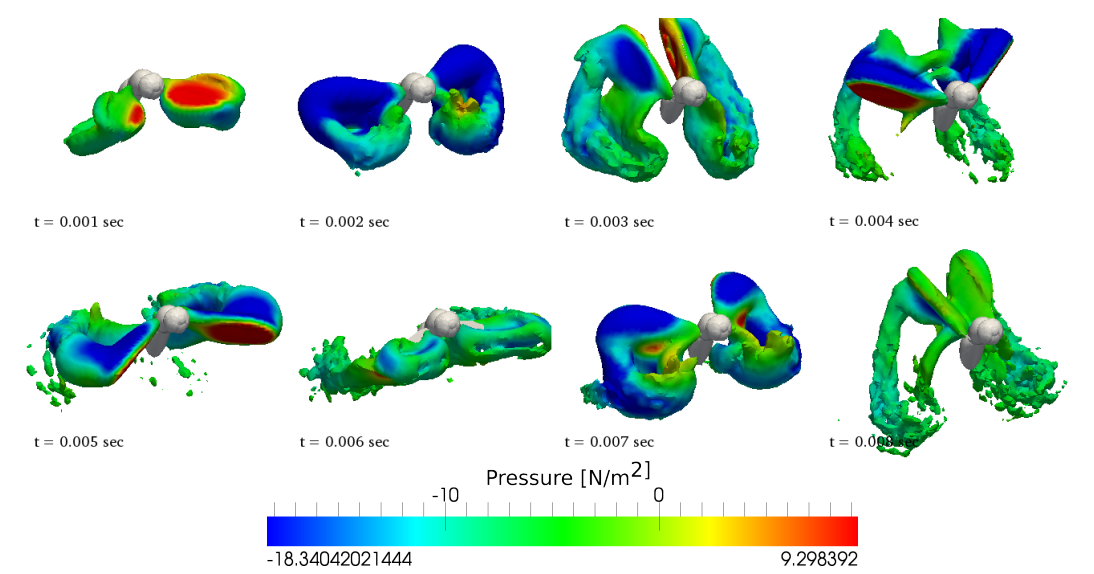

Fig. 11 Predicted isosurfaces of the vorticity magnitude $\left(4000 \mathrm{~s}^{-1}\right)$ coloured with the pressure value for different times of the stroke cycle.

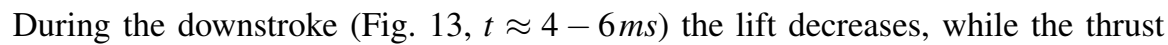
increases.

\section{Conclusions}

Our three-dimensional numerical simulations of the flow around the wings of freely flying fruit flies demonstrate highly-dynamic and complex vortical flow structures in insects. Detailed analyses of pressure- and shear forces suggest that the leading edge vortex, rotational circulation and wake capture tremendously contribute to aerodynamic force production in the insect. A comprehensive and comparative numerical analysis on the contribution of each of these force-enhancing mechanisms is still under investigation including an analysis on the impact of wing elasticity on forces 


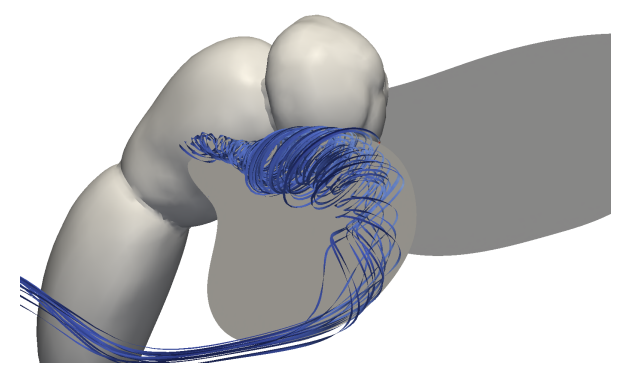

Fig. 12 Streamlines of LEV-induced flow downstream the leading edge during the wing's downstroke.

and moments. Moreover, a future goal of this project is to define similarity criteria of flight parameters that allow us to classify and group the maneuvers within the entire dataset. Eventually, these criteria are prerequisites in order to conduct profound statistics on wing kinematics and CFD data, and to understand the fluid dynamic features underlying the physical mechanisms of aerial maneuvering in insects.

\section{Acknowledgments}

This book chapter reports results from projects ,Experimental evaluation of forces and fluid structures during maneuvering flight of flies, using biomimetic robotics" and "Experimental quantification and numerical simulation of unsteady flow conditions during free flight maneuvers of insects" to CW and FOL, funded by grants LE905/8-2 and WA1510/13-2 of the DFG National-Priority-Program SPP1207 Impact of fluid flows in nature and technology.

\section{References}

[Demerec 1965] Demerec M (1965) Biology of Drosophila. New York and London: Hafner Publishing Company, 368-419

[Dickinson et al 1999] Dickinson MH, Lehmann F-O, Sane SP (1999) Wing rotation and the aerodynamic basis of insect flight. Science 284: 1954-1960

[Ellington et al 1996] Ellington CP, Van Berg CD, Willmott AP, Thomas ALR (1996) Leading-edge vortices in insect flight. Nature 384: 626-630

[Fontaine et al 2009] Fontaine EI, Zabala F, Dickinson MH, Burdick JW (2009) Wing and body motion during flight initiation in Drosophila revealed by automated visual tracking. J Exp Biol 212: 1307-1323

[Fry et al 2003] Fry SN, Sayaman R, Dickinson MH (2003) The aerodynamics of free-flight maneuvers in Drosophila. Science 300: 495-498 

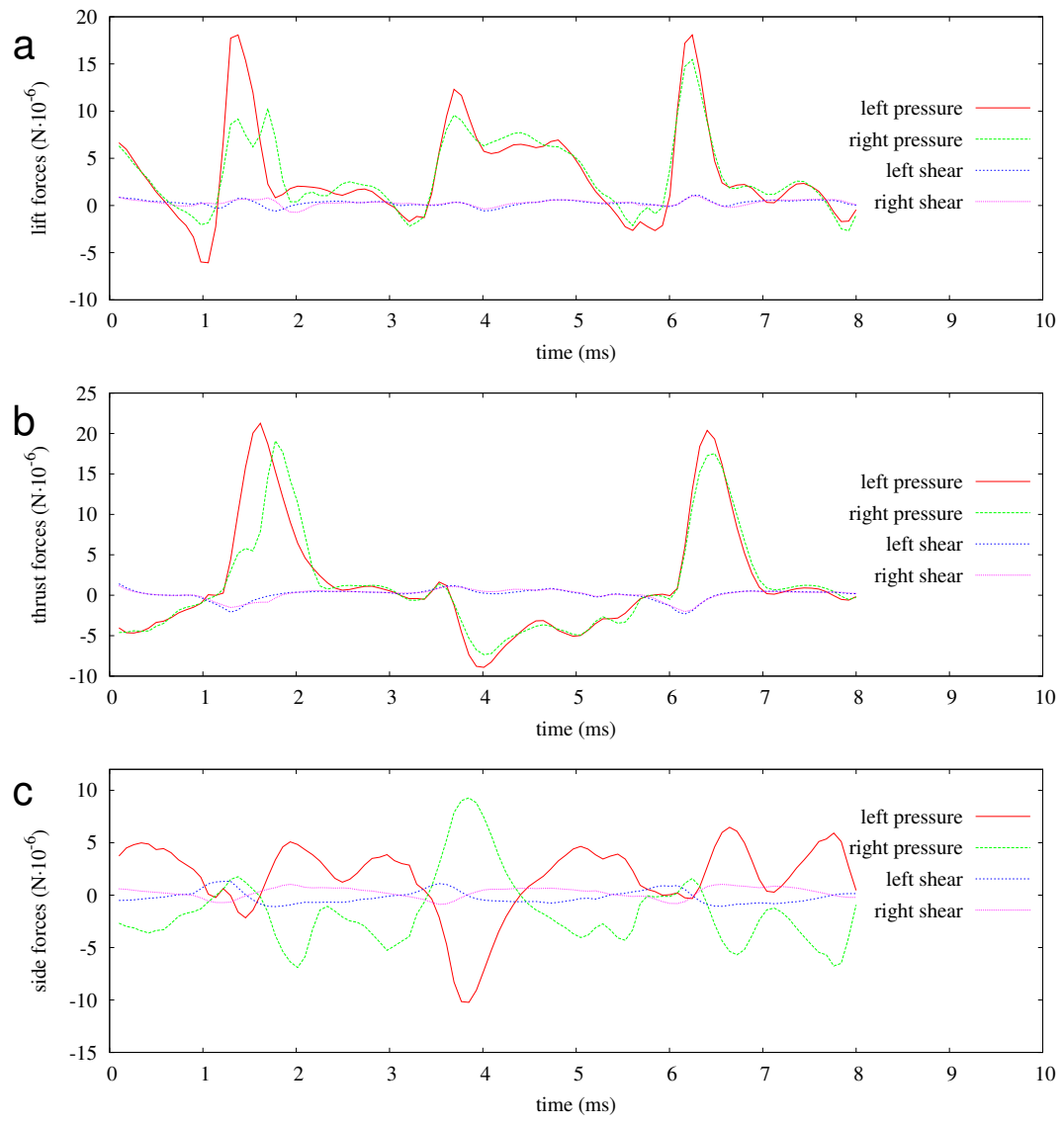

Fig. 13 Aerodynamic forces acting on the fruit fly's wings. a lift, $\mathbf{b}$ thrust and $\mathbf{c}$ lateral forces.

[Hedrick 2008] Hedrick TL (2008) Software techniques for two- and threedimensional kinematic measurements of biological and biomimetic systems. Bioinsp Biomim 3, doi:101088/1748-3182/3/3/034001

[Hedrick et al 2007] Hedrick TL, Usherwood JR, Biewener AA (2007) Low speed maneuvering flight of the rose-breasted cockatoo (Eolophus roseicapillus) II. Inertial and aerodynamic reorientation. J Exp Biol 210: 1912-1924

[2009] Hedrick TL, Cheng B, Deng X (2009) Wingbeat time and the scaling of passive rotational damping in flapping flight. Science 324: 252-255

[Hesselberg and Lehmann 2007] Hesselberg T, Lehmann F-O (2007) Turning behaviour depends on frictional damping in the fruit fly Drosophila J Exp Biol 210: 4319-4334

[Lehmann 2008] Lehmann F-O (2008) When wings touch wakes: understanding locomotor force control by wake-wing interference in insect wings. J Exp Biol 211: $224-233$ 
[Lehmann et al 2011] Lehmann F-O, Gorb S, Nasir N, Schützner P (2011) Elastic deformation and energy loss of flapping fly wings. J Exp Biol 214: 2949-2961

[Kliss et al 1989] Kliss M, Somps C, Luttges MW (1989) Stable vortex structures: A flat plate model of dragonfly hovering. J Theor Biol 136: 209-228

[Maybury and Lehmann 2004] Maybury WJ, Lehmann F-O (2004) The fluid dynamics of flight control by kinematic phase lag variation between two robotic insect wings J Exp Biol 207: 4707-4726

[Mronz and Lehmann 2008] Mronz M, Lehmann F-O (2008) The free-flight response of Drosophila to motion of the visual environment. J Exp Biol 211: 2026-2045

[2007] Ramamurti R, Sandberg WC (2007) A computational investigation of the three-dimensional unsteady aerodynamics of Drosophila hovering and maneuvering. J Exp Biol 210: 881-896

[Ristroph et al 2009] Ristroph L, Berman GJ, Bergou AJ, Wang ZJ, Cohen I (2009) Automated hull reconstruction motion tracking (HRMT) applied to sideways maneuvers of free-flying insects. J Exp Biol 212: 1324-1335

[Sane 2003] Sane S (2003) The aerodynamics of insect flight. J Exp Biol 206: 41914208

[Schützner and Lehmann 2007] Schützner P, Lehmann F-O (2007) High-speed analysis of wing and body motions in flying Drosophila using fluorescent markers. Proceedings of the International Symposium on Flying Insects and Robots, Monte Verit, Switzerland, August 12-17, 2007, 103-104

[Sun et al 2007] Sun M, Wang J, Xiong Y (2007) Dynamic flight stability of hovering insects. Acta Mech Sin 23: 231-246

[Wang 2000] Wang ZJ (2000) Two dimensional mechanism for insect hovering. Phys Rev Lett 85: 2216-2219 